\title{
Production under uncertainty and choice under uncertainty in the emergence of generalized expected utility theory
}

\author{
John Quiggin ${ }^{1}$ (DD
}

Accepted: 23 January 2022 / Published online: 28 February 2022

(C) The Author(s) 2022

\begin{abstract}
Interest in the foundations of the theory of choice under uncertainty was stimulated by applications of expected utility theory such as the Sandmo model of production under uncertainty. The development of generalized expected utility models raised the question of whether such models could be used in the analysis of applied problems such as those involving production under uncertainty. Finally, the revival of the state-contingent approach led to the recognition of a fundamental duality between choice problems and production problems.
\end{abstract}

Keywords Production - Choice - Uncertainty

The field of generalized expected utility theory had its beginnings with the classic paper of Allais (1953), proposing counterexamples to the expected utility (EU) theory of Von Neumann and Morgenstern (1944). Although this paper was wellknown, and frequently cited, over the next 3 decades, it was not until the late 1970s that Allais' critique became the basis of a substantial research program. The statecontingent approach to production under uncertainty has had a rather similar history.

When economists were first embarking on the study of problems involving uncertainty, Arrow (1953) and Debreu (1952) developed the elegantly simple idea of state-contingent commodities. The project of developing a rigorous general equilibrium theory had already led to the notion of differentiating commodities by their time and place of delivery. It was a relatively small step to deal with uncertainty through the notion of state-contingent commodities; that is, commodities whose delivery is contingent on the occurrence of a particular state of nature. Once this connection was made, all the tools developed for a non-stochastic world could be applied almost effortlessly to decision-making under uncertainty. In

John Quiggin

j.quiggin@uq.edu.au

1 Department of Economics, University of Queensland, Brisbane 4072, Australia 
Debreu's words, the notion of a state-contingent commodity “... allows one to obtain a theory of uncertainty free from any probability concept and formally identical with the theory of certainty ...” (p. 98). Yaari (1969) developed this point further showing how notions of comparative risk aversion could be developed in a purely state-contingent framework, without any necessary reliance on probability distributions.

This should have been welcome news to economists. It means that tools honed in other areas can be used to analyze decision-making under uncertainty. Even more force was added to the argument by Hirshleifer's (1965) demonstration of the analogy between the insights obtained from a state-contingent interpretation of uncertainty and the way in which Fisher's (1930) treatment of time-preference had demystified the concept of production and consumption over time. Unfortunately, except in fairly restricted areas of economic theory, this pathbreaking insight was ignored in the analysis of production decisions under uncertainty.

Instead, initial analyses of the firm under uncertainty were largely undertaken using what was, in effect, a stochastic production function approach. This approach appeared to be simpler than the general state-contingent approach and seemed to allow exploitation of the analogy between production problems involving uncertainty and portfolio allocation problems. In fact, however, the apparent simplicity of the approach can be preserved only if attention is restricted to scalar choice sets.

Moreover, the analytical tools applied to the problem of the firm under uncertainty were derived under the assumption of expected-utility maximization. A partial extension to the case of rank-dependent expected utility was possible (Quiggin 1991), but attempts to undertake comparative static analysis in any more general context encountered significant difficulties (Machina 1989). However, the expected utility hypothesis is largely redundant in the analysis of state-contingent production (Chambers and Quiggin 2000; Quiggin and Chambers 2001).

This paper presents a personal view of the interaction between the analysis of choice under uncertainty and the analysis of production under uncertainty. As regards the former, the main focus is on rank-dependent models, particularly the anticipated utility model of Quiggin (1979, 1981, 1982a), Choquet expected utility (Schmeidler 1989) and cumulative prospect theory (Tversky and Kahneman 1992). A common thread linking all of these models is the work of Peter Wakker in developing rigorous axiomatic derivations (Quiggin and Wakker 1994; Wakker and Tversky 1993) and in exposition of the intuition underlying the theory and the relationships between them (Diecidue and Wakker 2001; Wakker 1989, 1990).

\section{Generalized expected utility theory}

Over the course of the 1970s, the expected-utility hypothesis came under increasing attack. The long-neglected and much-misinterpreted criticisms of Allais (1953) were reinforced by new empirical evidence, which demonstrated the robustness of the 'Allais paradox'. Other long-standing problems, such as the co-existence of insurance and lottery gambling (Friedman and Savage 1948) received renewed attention. Attempts to elicit utility functions using the assumption of preferences 
'linear in the probabilities' produced systematic violations of the consistency assumptions of expected utility theory. A number of attempts were made to develop generalizations of expected utility theory that could account for the Allais paradox and related phenomena such as the common ratio effect.

One approach which fell into the category of 'fruitful error' was that of Handa (1977), who proposed a model based on the idea of probability weighting. Unfortunately, Handa's model implied violation of statewise dominance, that is, the requirement that if one prospect yields a better outcome than another in every state of the world, it must be preferred. However, Handa's proposed axioms include the weaker property of preservation of first-order stochastic dominance. ${ }^{1}$

The Journal of Political Economy (JPE) was deluged with comments pointing out this error, of which the most elegant, and the only one published, was that of Fishburn (1978). Other contributions came from Mark Machina, then a graduate student at MIT, and from me, then a civil servant with the Bureau of Agricultural Economics (BAE) in Canberra, Australia, and working on an Honours thesis focusing on the Sandmo model.

Shortly afterwards, the classic paper of Kahneman and Tversky (1979) introduced the world to 'prospect theory' which became the most widely used alternative to expected utility theory. The key idea was that of a reference point, along prospects to be separated into gains and losses, with the idea that risk aversion would prevail for gains, and risk seeking for losses. Along with this and other changes to the expected utility model, Kahneman and Tversky proposed a model of probability weighting broadly similar to that of Handa. The problem of first-order stochastic dominance was dealt with by the inclusion of an 'editing phase' in which dominated prospects are eliminated from choice sets.

It was thinking about how to correct the Handa model that led me to develop rank-dependent utility, which I initially described as 'anticipated utility' (AU). ${ }^{2}$ The crucial idea was that the weight associated with an outcome should depend on its rank in the distribution as well as on its probability. As was observed in Quiggin (1982a):

The following example illustrates further the notion that equally probable events should not necessarily receive the same weight. Suppose an individual's normal wage income is uniformly distributed over a range from

\footnotetext{
${ }^{1}$ First-order stochastic dominance requires that if, for any $x$, one prospect $y$ gives at least as high probability of an outcome equal to or better than $x$ than does an alternative prospect $y^{\prime}$ then $y$ must be preferred to $y^{\prime}$. In formal terms

$1-F_{y}(x) \geq 1-F_{y^{\prime}}(x) \forall x \Rightarrow y \succeq y^{\prime}$

where $F$ is the cumulative distribution function.

2 The term 'anticipated utility' was simply intended to mean 'generalized expected utility', since 'anticipated' is a more general synonym of 'expected'. At the time the term 'anticipated utility' was coined, I assumed that no other generalized expected utility model, consistent with first-order stochastic dominance, was possible. In fact, this is only true if a functional separation between probabilities and utilities is required. When it became apparent that 'anticipated utility' was only one of many 'generalized expected utility' models, I adopted the term 'rank-dependent expected utility' proposed by Chew and Epstein (1989).
} 
$\$ 20,000.01$ to $\$ 21,000.00$. There is also a $1 / 100,000$ chance that the person will win a contest for which the prize is a job paying $\$ 1 \mathrm{~m}$ a year. The probability of receiving any specified income in the relevant range $\$ 20,000.01$ to $\$ 21,000.00$ (e.g. $\$ 20,439.72$ ) is also $1 / 100,000$. Nevertheless, it seems reasonable that the extreme outcome will not be weighted in the same way as an intermediate outcome such as $\$ 21,439.72$.

The idea that extreme outcomes might be overweighted led to the anticipated utility (AU) or rank-dependent expected utility (RDU) model. Using the notation of Quiggin and Wakker (1994), the model may be described as follows. Consider a set of outcomes $X$ and a set $Y$ of prospects, consisting of all probability distributions over $X$ with finite support. A typical element of $Y$ is a pair $\left\{\left(x_{1}, x_{2} \ldots x_{n}\right) ;\left(p_{1}, p_{2} \ldots p_{n}\right)\right\}=\{\mathbf{x} ; \mathbf{p}\}$ yielding outcome $x_{j}$ with probability $p_{j}$. By $\succeq$ denote a binary preference relation over $Y$, with associated strict preference $\succ$ and indifference $\sim$ and use $\succeq$ also to represent the induced ordering on $X$. The crucial innovation was to focus attention on rank-ordered prospects, in which $x_{1} \leq x_{2} \leq \cdots \leq x_{n}$. RDU holds if the relation $\succeq$ can be represented by a function $V: Y \rightarrow \mathbb{R}$ of the form

$$
V\left\{\left(x_{1}, x_{2} \ldots x_{n}\right) ;\left(p_{1}, p_{2} \ldots p_{n}\right)\right\}=\sum_{i=1}^{n}\left(f\left(\sum_{j=1}^{i} p_{j}\right)-f\left(\sum_{j=1}^{i-1} p_{j}\right)\right) U\left(x_{i}\right)
$$

for a utility function $U: X \rightarrow \mathbb{R}$ and a non-decreasing transformation function $f$ : $[0,1] \rightarrow[0,1]$ with $f(0)=0, f(1)=1$. Note that, with the convention $\sum_{j=1}^{0} p_{j}$

$$
\sum_{i=1}^{n}\left(f\left(\sum_{j=1}^{i} p_{j}\right)-f\left(\sum_{j=1}^{i-1} p_{j}\right)\right)=f(1)=1
$$

so that the decision weights $w_{i}=f\left(\sum_{j=1}^{i} p_{j}\right)-f\left(\sum_{j=1}^{i-1} p_{j}\right)$ sum to 1 .

With this representation, $\mathrm{AU}$ is the special case where $f\left(\frac{1}{2}\right)=\frac{1}{2}$.

The functional form associated with the rank-dependent model was independently rediscovered on several separate occasions, with quite different motivating arguments. Examples include Allais (1987), Yaari (1987) and, in the context of social choice, Weymark (1981) and Ebert (1988). Thus, the model represents an interesting example of the theory of multiple discoveries, put forward by Merton (1973).

Tversky and Kahneman (1992) modified the original prospect theory to include rank-dependent probability, yielding cumulative prospect theory. Cumulative prospect theory was axiomatised by Wakker and Tversky (1993). Kahneman received the 2002 Nobel Memorial Prize in Economic Sciences for this work, and Tversky a rare posthumous mention. Wakker (2010) provides the best guide to the literature.

The rank-dependent model was successful in resolving many of the concerns raised by critics of Expected Utility Theory. Quiggin (1982a, 1982b) showed how AU could resolve the paradoxes noted by Friedman and Savage (1948) and Allais 
(1953). Wakker and Deneffe (1996) show how to take rank-dependent probability weighting into account when eliciting utility functions over outcomes.

\subsection{Axioms for anticipated utility and related models}

Quiggin $(1979,1982 b)$ proposed an axiomatic basis for anticipated utility, in which the standard independence axiom was restricted to apply only to 50-50 mixtures between rank-ordered prospects. This set of axioms implied the restriction $f(1 / 2)=1 / 2$, dropped in subsequent formulations of RDU. Even with this restriction, the derivation of the AU model from the axioms was incorrect. A more accurate statement is given by Quiggin and Wakker (1994). ${ }^{3}$ Quiggin and Wakker (1994) propose the following structural restrictions, which are a modified version of those in Quiggin (1982b).

Axiom 1: (Completeness): The binary relation $\succeq$ is a complete weak order

Axiom 2: (Dominance) 2'a: If $p^{\prime} \geq p$, then $\left\{\left(x_{1}, x_{2}\right) ;\left(1-p^{\prime}, p^{\prime}\right)\right\} \succeq$ $\left\{\left(x_{1}, x_{2}\right) ;(1-p, p)\right\}$

$2^{\prime}$ b: $\left\{\left(x_{1}^{\prime}, x_{2}^{\prime}\right) ;\left(\frac{1}{2}, \frac{1}{2}\right)\right\} \succeq\left\{\left(x_{1}, x_{2}\right) ;\left(\frac{1}{2}, \frac{1}{2}\right)\right\}$ whenever $x_{2}^{\prime} \succeq x_{2}, x_{1}^{\prime} \succeq x_{1}$ where the former preference is strict if one of the latter two is strict.

Axiom 3: (Continuity) If $x_{1} \preceq x_{2} \preceq x_{3} \exists X$, then there exists $p^{*}$ such that $x_{2}^{\sim}\left\{\left(x_{1}, x_{3}\right) ;\left(1-p^{*}, p^{*}\right)\right\}$.

Axiom 4: (Independence) Whenever $\quad x^{\sim}\{\mathbf{x} ; \mathbf{p}\}, x^{\prime} \sim\left\{\mathbf{x}^{\prime} ; \mathbf{p}^{\prime}\right\}, \quad$ and $c_{i}^{\sim}\left\{\left(x_{i}, x_{i}^{\prime}\right) ;\left(\frac{1}{2}, \frac{1}{2}\right)\right\}$ for all $i$, then $\{\mathbf{c} ; \mathbf{p}\} \sim\left\{\left(x, x^{\prime}\right) ;\left(\frac{1}{2}, \frac{1}{2}\right)\right\}^{4}$

Axioms $2^{\prime}$ a and $2^{\prime}$ b replace a weaker axiom proposed by Quiggin (1982a), which is insufficient to ensure that $f$ is monotonic, or that it is bounded above by 1 . Axiom 4 is the modification of the von Neumann-Morgenstern independence axiom proposed by Quiggin (1982a). Axioms for the general case of RDU are given by Quiggin and Wakker (1994).

\subsection{The state preference approach}

The literature on generalized expected utility arose mainly out of debates about the von Neumann-Morgenstern model of expected utility, in which the objects of preference were probability distributions. In particular, the counterexamples proposed by Allais, and the violations of EU predictions elicited by Kahneman and Tversky were all developed in this context.

The von Neumann-Morgenstern framework took probabilities as primitive. In contrast, Savage (1954) began with preferences over acts, represented as mappings from a set of states of nature (presumed to be exhaustive and mutually exclusive) to a set of outcomes. Savage presented axioms which simultaneously implied the existence of both well-defined subjective probabilities for states and a utility

\footnotetext{
3 Despite the alphabetical ordering, Wakker was the primary author of this paper. I was very pleased that the mistakes in my 1982 paper produced my only opportunity, so far, for such a co-authorship.

${ }^{4}$ Since the ordering of outcomes is irrelevant in the case of a 50-50 bet, we make no assumption about the ordering of $x$ and $x^{\prime}$ here.
} 
function (unique up to an affine transformation) over outcomes, such that the preferred element of any set of acts was that which maximized expected utility.

Although the RDU model dealt with preferences over probability distributions, the weight placed on any given state depended on the ranking of the outcome associated with that state under a given act. Particularly in cases of comonotonic acts, where the ranking of states is the same for both acts, the effect is to pay more attention to the state-act pair associated with a given probability distribution. Diecidue and Wakker (2001) use the idea of comonotonicity as an intuitive basis for RDU.

The Choquet Expected Utility model developed by Schmeidler (1989) extended the idea of rank dependence to the Savage model. Wakker (1990) showed that the Choquet expected utility model of is equivalent to RDU if and only if preferences are probabilistically sophisticated in the sense of Machina and Schmeidler (1992), that is, consistent with first-order stochastic dominance for some probability distribution.

\section{Production under uncertainty}

The fact that production is subject to uncertainty is central to economics. If the outcomes of production decisions, defined broadly to include household production, were perfectly predictable, there would be no need for insurance or finance, and no reason for the occurrence of macroeconomic fluctuations. Yet, the theory of production under uncertainty remains underdeveloped. Little account has been taken of theoretical advances in the theory of choice under uncertainty.

The first formal treatment of production under uncertainty was the general equilibrium analysis put forward independently by Arrow (1953) and Debreu (1952). Using the notion of state-contingent commodities, Arrow and Debreu showed that the proof of the existence of competitive equilibrium, based on the Kakutani fixed-point theorem carried over, in a formal sense, to the case of uncertainty. The Arrow-Debreu analysis forms the basis of modern finance theory. However, as far as analysis of the behavior of firms under uncertainty is concerned, little progress was made. Tobin's (1969) assessment that state-preference theory was 'graceful but empty' remained apposite three decades later.

\subsection{The state-contingent and parameterized distribution frameworks}

The distinction between state-contingent and distribution function representaion, already discussed in the context of generalized expected utility theory, plays a central role in the theory of production under uncertainty.

Much analysis, including early formulations of the anticipated utility model, deals with choice over families of probability distributions or, more generally, cumulative distribution functions, that is, monotone increasing mappings from an ordered outcome space $X$ to the unit interval $[0,1]$. In the typical case where these families are indexed by a single parameter or a small set of parameters, this approach may be referred to as the parametrized distribution representation of 
choice under uncertainty. The stochastic production function model, in which each choice of input $z$ is associated with the random output $f(z, \theta)$ is an example of the parametrized distribution representation.

In the parametrized distribution representations, the probabilities associated with the different states are treated as objectively given (although amenable to manipulation by altering the action or input vector). The critical question is: How can these probabilities be defined and observed?

An alternative view, which may be traced back to the work of de Finetti (1931) and Savage (1954), is that probabilities used in economic decision-making are inherently subjective. Consequently, probabilities can only be inferred from the observed behavior of decision-makers, and hence they are inseparably tied to beliefs and preferences. A logical consequence of this position is the recognition that any statement about subjective probabilities is ultimately a statement about the decisionmaker's beliefs and preferences. Therefore, intermingling probabilities with the technology of production, as is done in the parametrized distribution formulation, represents an important logical confusion.

This view leads to the adoption of a state-contingent representation of choice under uncertainty, in which the primitive objects of choice are acts, regarded as mappings from a set of states of nature to a set of outcomes. More formally, we are concerned with preferences over state-contingent outcome vectors, represented as mappings from a state space $\Omega$ to an outcome space $X \subseteq \Re$. Preferences over $X^{\Omega}$ are given by a total ordering denoted notationally by $\succ$. A preference function is a mapping $W: X^{S} \rightarrow \Re$ such that $W(\mathbf{x})>W\left(\mathbf{x}^{\prime}\right)$ if and only if $\mathbf{x} \succ \mathbf{x}^{\prime} . W$ is assumed everywhere continuous and nondecreasing.

Since the state-contingent framework is capable of incorporating preferences that may or may not be probabilistically sophisticated, it is more general than the parameterized distribution framework. Over time, it has become apparent that the state-contingent approach is analytically more tractable, and that the existence of well-defined subjective probabilities is most appropriately regarded as a restriction on the functional form of a preference representation, analogous to additive separability in preferences over non-stochastic consumption bundles.

\section{The Sandmo model}

As noted above, the Arrow-Debreu representation of production under uncertainty was not developed beyond the point required to establish existence theories for general equilibrium. Microeconomic analysis of production under uncertainty began with Sandmo (1971), using a parametrized distribution function approach.

Sandmo drew on yet another multiple discovery, that of the Arrow-Pratt measure of risk aversion (Pratt 1964; Arrow 1965). Sandmo considered the simple case of a firm producing a single good, here denoted $z$, at a cost $C(z)$ and facing a random, competitively determined price $P$. The owner of the firm is a risk-averse expected utility maximizer who seeks to maximize the objective function 


$$
\max _{x} W=E[u(P z-C(z))] .
$$

In the linear case where $C(z)=C z$, this problem is exactly analogous to the twoasset portfolio problem, where the safe asset returns zero and the risky asset yields the random return $(P-C)$. Sandmo extended a number of the results known to hold for the two-asset portfolio problem to the more general case of the firm under price uncertainty. In particular, he showed that given decreasing absolute risk aversion, an increase in mean price would result in an increase in output.

Equally importantly, Sandmo left a number of loose ends and conjectures to stimulate subsequent researchers. Coes (1977) confirmed Sandmo's conjecture that, assuming decreasing absolute risk aversion, a multiplicative increase in price risk would lead to a reduction in output. Quiggin (1982b) derived conditions for the existence of a finite optimum (most of these conditions had been established earlier, for a more general formulation of the decision problem, by Bertsekas (1974).)

Other writers generalized Sandmo's analysis. An easy generalization is to reinterpret the model as one in which the firm faces technological uncertainty. Replace $P$ by a random yield variable $\theta$ and interpret $z$ as an input variable, such as the area of land devoted to a crop. A more substantial change is to allow for a general stochastic production function $x(z, \theta)$ where $\theta$ can be interpreted as an input contributed by 'Nature' such as rainfall. The objective function, therefore, becomes:

$$
\max _{x} V=E[u(P x(z, \theta)-w z)] .
$$

where $w$ is the price of the market input $z$. Feder (1977) analyzed the comparative static problems of choice problems involving such general objective functions. Epstein (1978) extended this analysis to the case of multiple inputs. This allows for a more flexible technology, but not as flexible as the general state-contingent technology considered below.

This analysis took place under the assumption of expected utility maximization. Quiggin (1991) showed that all of the main results could be extended to the case of RDU. The key insight here is that, in problems of the general class considered by Feder, maximization of RDU may be considered as maximization of EU with respect to a transformed probability distribution.

\section{State-contingent production}

In the state-contingent framework, a production decision may be regarded as an act which requires a non-stochastic input $z$, and yields an output $x$ in every state of nature. It is straightforward to reinterpret the stochastic production function model in state-contingent terms. If the state space is $S$, the set of acts may be identified with the set of possible input choices $z$, each of which produces the outcome $(z, \mathbf{x})$ where $\mathbf{x} \in \Re^{S}$, and $x_{s}$ is the output realized if state $s \in S$ occurs. This is analogous to a multi-output production function.

A deeper insight, due to Arrow (1953) and Debreu (1952), is that the modern approach to producer and consumer theory, based on sets and correspondences 
rather than functions, may be applied without modification, to problems involving uncertainty. Arrow and Debreu used this insight to extend general equilibrium theory to encompass the case of uncertainty.

However, until the development of the modern approach to production analysis, based on the duality between prices and quantities in optimization problems (Shephard 1970; McFadden 1978; Chambers 1988), few tools were available to permit the use of set-theoretic representations of production technologies in the analysis of firm behavior. Consequently, most applied analysis of firm behavior was based on the older idea of a production function. And when issues relating to firmlevel stochastic production began to be seriously considered by theorists, they naturally based their analysis on the related notion of a stochastic production function. The widespread success of the axiomatic approach in creating the superstructure of duality theory and its many applications, however, suggests the possibility of an extension of this analysis to a state-contingent production technology as in the work of Arrow and Debreu.

The groundwork for such an extension is presented by Chambers and Quiggin (2000). Chambers and Quiggin represent the production technology in the form of an input correspondence which maps matrices of state-contingent outputs into sets of inputs that are capable of producing that state-contingent output matrix. Formally, it is defined by:

$$
Z(\mathbf{x})=\left\{\mathbf{z} \in \Re_{+}^{N}: \mathbf{z} \text { can produce } \mathbf{x} \in \Re_{+}^{M x S}\right\} .
$$

Intuitively, $Z(\mathbf{x})$, typically referred to as the input set, is identified with everything on or above an isoquant for the state-contingent technology.

We may derive the cost function

$$
C(\mathbf{x}, \mathbf{w})=\min \{\mathbf{w} \bullet \mathbf{z}: \mathbf{z} \in Z(\mathbf{x})\}
$$

if there exists a feasible input vector capable of producing $\mathbf{x}$, and $\infty$ otherwise.

A trivial but crucial observation is that, for given $\mathbf{w}$, the revenue-cost function is, like the preference function $W$, a mapping from $\Re^{S}$ to $\Re$. Hence, for any property of the preference function, an analogous property may be defined for the revenue-cost function, and vice versa. The analysis of risk premiums yields some important applications of this observation.

\section{Risk premiums in choice and production}

Once the analogy between state-contingent income or output vectors and multicommodity consumption or production bundles is recognized, a wide range of analytical tools become available. In particular, the concept of a risk premium, which has proved valuable in analysis using the expected-utility model, may be generalized using Luenberger's (1992) benefit function for the preference structure. The benefit function, $B: \Re \times X^{S} \rightarrow \Re$, is defined for $\mathbf{g} \in \Re^{S}$ by: 


$$
B(w, \mathbf{x})=\max \{\beta \in \Re: W(\mathbf{x}-\beta \mathbf{1}) \geq w\}
$$

if $W(\mathbf{x}-\beta \mathbf{1}) \geq w$ for some $\beta$, and $-\infty$ otherwise. Similarly, concepts of relative risk aversion may be analyzed using the Shephard (1953) and Malmquist (1953) distance function $D: \Re_{+}^{S} \times \Re \rightarrow \Re_{+}$defined by:

$$
D(\mathbf{x}, w)=\sup \{\lambda>0: W(\mathbf{x} / \lambda) \geq w\} \quad y \in \Re_{+}^{S} .
$$

For any expectation operator $E$ define the absolute risk premium:

$$
\begin{aligned}
r(\mathbf{x}) & =\max \{c: W((E[\mathbf{x}]-c) \mathbf{1}) \geq W(\mathbf{y})\} \\
& =B(W(\mathbf{y}), E[\mathbf{x}] \mathbf{1}),
\end{aligned}
$$

and the relative risk premium:

$$
\begin{aligned}
v(\mathbf{x}) & =\sup \{\lambda>0: W(E[\mathbf{x}] \mathbf{1} / \lambda) \geq W(\mathbf{x})\} \\
& =D(E[\mathbf{x}] \mathbf{1}, W(\mathbf{x})) .
\end{aligned}
$$

For any $\mathbf{y}$ and $W$, define the certainty equivalent:

$$
e(\mathbf{x})=\inf \{c>0: W(c \mathbf{1}) \geq W(\mathbf{x})\}
$$

and observe:

$$
\begin{aligned}
r(\mathbf{x}) & =E[\mathbf{x}]+B(W(\mathbf{x}), \mathbf{0}) \\
& =E[\mathbf{x}]-e(\mathbf{x}) . \\
v(\mathbf{y}) & =E[\mathbf{x}] D(\mathbf{1}, W(\mathbf{x})) \\
& =E[\mathbf{x}] / e(\mathbf{x}) .
\end{aligned}
$$

\subsection{Production risk}

An exactly dual analysis applies in the case of production risk. Just as a risk-averse individual will pay a premium in each state to ensure the certainty outcome, achieving the certainty outcome may prove costly. That is, typically, it should cost more to remove production uncertainty and produce the same non-stochastic output in each state than to allow for stochastic production.

For the cost function, $C(\mathbf{x}, \mathbf{w})$, and $\mathbf{r} \in \Re_{+}^{S}$, define the certainty equivalent output, denoted by $e^{c}(\mathbf{x}, \mathbf{w}) \in \Re_{+}$, as the maximum non-stochastic output that can be produced at cost $C(\mathbf{w}, \mathbf{r}, \mathbf{P})$, that is,

$$
e^{c}(\mathbf{x}, \mathbf{w})=\sup \left\{e: C\left(e \mathbf{1}^{S}, \mathbf{w}\right) \leq C(\mathbf{x}, \mathbf{w})\right\},
$$

where $\mathbf{1}^{S}$ is the $S$-dimensional unit vector. By analogy with the risk premium used in the theory of consumer choice, define the production-risk premium as the difference between mean revenue and the certainty equivalent revenue. Notationally, the production risk premium is defined by 


$$
p(\mathbf{x}, \mathbf{w})=E[\mathbf{x}]-e^{c}(\mathbf{x}, \mathbf{w})
$$

and satisfies:

$$
\begin{aligned}
C(\mathbf{x}, \mathbf{w}) & =C\left(\overline{\mathbf{x}}-p(\mathbf{x}, \mathbf{w}) \mathbf{1}^{\mathbf{S}}, \mathbf{w}\right) \\
& =C\left(e^{c}(\mathbf{x}, \mathbf{w}) \mathbf{1}^{S}, \mathbf{w}\right) .
\end{aligned}
$$

where $\overline{\mathbf{x}} \in \Re_{+}^{S}$ denotes the vector with $E[\mathbf{x}]$ occurring in each state.

The certainty equivalent revenue and the production risk premium are alternative characterizations of the technology. Formally, this can be verified by noting that the certainty equivalent revenue is a nondecreasing transformation of revenue-cost.

\section{Concluding comments}

The interaction between production theory and consumer theory has been very important in the development of the theory of economics under certainty. Much less interaction has been evident in the theory of choice under uncertainty. As this paper has shown, however, both analysis of production problems involving uncertainty and the inherent logic of the rank-dependent expected utility model lead naturally to a focus on state-contingent representations of uncertainty.

To a large extent, this paper has followed my own intellectual path through the theory of choice and production under uncertainty, beginning with exposure to the pioneering work of Allais on decision theory and Sandmo on production under uncertainty. The development of my ideas has repeatedly been intertwined with, and enhanced by the work of Peter Wakker, who is an almost exact contemporary of mine. On the occasion of his 65 th birthday, I am glad to have the opportunity to recognize his contributions.

Funding Open Access funding enabled and organized by CAUL and its Member Institutions.

Open Access This article is licensed under a Creative Commons Attribution 4.0 International License, which permits use, sharing, adaptation, distribution and reproduction in any medium or format, as long as you give appropriate credit to the original author(s) and the source, provide a link to the Creative Commons licence, and indicate if changes were made. The images or other third party material in this article are included in the article's Creative Commons licence, unless indicated otherwise in a credit line to the material. If material is not included in the article's Creative Commons licence and your intended use is not permitted by statutory regulation or exceeds the permitted use, you will need to obtain permission directly from the copyright holder. To view a copy of this licence, visit http:// creativecommons.org/licenses/by/4.0/.

\section{References}

Allais, M. (1953). Le comportement de l'homme rationnel devant le risque: Critique des axioms et postulats de l'école Americaine. Econometrica, 21, 503-46.

Allais, M. (1987) The general theory of random choices in relation to the invariant cardinal utility function and the specific probability function: The $(\mathrm{U}, \theta)$ model - A general overview. In Risk, Decision and Rationality, (Ed, Munier, B.) pp. 233-89.

Arrow, K. (1953). Le rôle des valeurs boursiers pour la repartition la meillure des risques. Cahiers du Seminair d'Economie, CNRS. 
Arrow, K. (1965). Aspects of the theory of risk-bearing. Yrjo Jahnsson Lecture.

Bertsekas, D. (1974). Necessary and sufficient conditions for the existence of an optimal portfolio. Journal of Economic Theory, 8, 235-47.

Chambers, R. G. (1988). Applied production analysis: A dual approach. Cambridge University Press.

Chambers, R. G., \& Quiggin, J. (2000). Uncertainty, production, choice and agency: The state-contingent approach. Cambridge University Press.

Chew, S. H., \& Epstein, L. (1989). Axiomatic rank-dependent means. Annals of Operations Research, 19, 207-40.

Choquet, G. (1953-4). Theory of capacities. Annales Institut Fourier,5, 131-295.

Coes, D. (1977). Firm output and changes in uncertainty. American Economic Review, 67, $249-51$.

de Finetti, B. (1931). Sul significato soggettivo della probabilita. Fundamenta Mathematicae, 17, 298-329.

Debreu, G. (1952). A social equilibrium existence theorem. Proceedings of the National Academy of Sciences, 38, 886-93.

Diecidue, E., \& Wakker, P. P. (2001). On the intuition of rank-dependent expected utility. Journal of Risk and Uncertainty, 23(3), 281-98.

Ebert, U. (1988). Rawls and Bentham reconciled. Theory and Decision, 24, 215-223.

Epstein, L. (1978). Production flexibility and the behaviour of the competitive firm under price uncertainty. Review of Economic Studies, 45(2), 251-261.

Feder, G. (1977). The impact of uncertainty in a class of objective functions. Journal of Economic Theory, 16, 504-12.

Fishburn, P. (1978). On Handa's new theory of cardinal utility' and the maximization of expected return. Journal of Political Economy, 86, 321-4.

Fishburn, P. (1988). Nonlinear preference and utility theory. Johns Hopkins.

Fisher, I. (1930). The theory of interest. Yale University Press.

Friedman, M., \& Savage, L. J. (1948). The utility analysis of choices involving risk. Journal of Political Economy, 56(4), 279-304.

Gilboa, I. (1987). Expected utility with purely subjective non-additive probabilities. Journal of Mathematical Economics, 16, 65-88.

Handa, J. (1977). Risk, probabilities and a new theory of cardinal utility. Journal of Political Economy, $85,97-122$

Hirshleifer, J. (1965). Investment decisions under uncertainty: Choice-theoretic approaches. Quarterly Journal of Economics, 79(4), 509-36.

Kahneman, D., \& Tversky, A. (1979). Prospect theory: an analysis of decision under risk. Econometrica, 47(2), 263-91.

Luenberger, D. G. (1992). Benefit functions and duality. Journal of Mathematical Economics, 21, 461-481.

Machina, M. (1989). Comparative statics and non-expected utility preferences. Journal of Economic Theory, 47(2), 393-405.

Machina, M., \& Schmeidler, D. (1992). A more robust definition of subjective probability. Econometrica, 60(4), 745-80.

Malmquist, S. (1953). Index numbers and indifference surfaces. Trabajos de Estatistica, 4, $209-242$.

McFadden, D. (1978). Cost, revenue, and profit functions. In M. Fuss \& D. McFadden (Eds.), Production economics: A dual approach to theory and applications. North Holland.

Merton, R. (1973). The sociology of science. University of Chicago Press.

Pratt, J. (1964). Risk aversion in the small and in the large. Econometrica, 32, 122-36.

Quiggin, J. (1979). The multi-product firm under price uncertainty, unpublished Honours thesis, Australian National University.

Quiggin, J. (1981). Risk perception and risk aversion among Australian farmers. Australian Journal of Agricultural Economics, 25, 160-9.

Quiggin, J. (1982). A note on the existence of a competitive optimum. Economic Record, 55, 174-6.

Quiggin, J. (1982). A theory of anticipated utility. Journal of Economic Behavior and Organization, 3, 323-43.

Quiggin, J. (1991). Comparative statics for rank-dependent expected utility theory. Journal of Risk and Uncertainty, 4, 339-50.

Quiggin, J., \& Chambers, R. G. (1998). Risk premiums and benefit measures for generalized expected utility theories. Journal of Risk and Uncertainty, 17, 121-37. 
Quiggin, J., \& Chambers, R. G. (2000). The firm under price and output uncertainty: The state-theoretic approach. Journal of Risk and Uncertainty., 22(1), 5-20.

Quiggin, J., \& Wakker, P. (1994). The axiomatic basis of anticipated utility: A clarification. Journal of Economic Theory, 64(2), 486-99.

Sandmo, A. (1971). On the theory of the competitive firm under price uncertainty. American Economic Review, 61, 65-73.

Savage, L. (1954). Foundations of statistics. Wiley.

Schmeidler, D. (1989). Subjective probability and expected utility without additivity. Econometrica, 57, 571-87.

Shephard, R. W. (1953). Cost and production functions. Princeton University Press.

Shephard, R. W. (1970). Theory of cost and production functions. Princeton University Press.

Tobin, J. (1969). Comment on Borch and Feldstein. Review of Economic Studies, 36, 13-14.

Tversky, A., \& Kahneman, D. (1992). Cumulative prospect theory: An analysis of attitudes towards uncertainty and value. Journal of Risk and Uncertainty, 5(3), 297-323.

Von Neumann, J., \& Morgenstern, O. (1944). Theory of games and economic behavior. Princeton University Press.

Wakker, P. (1989). 'Transforming probabilities without violating stochastic dominance. In E. Roskam (Ed.), Mathematical psychology in progress (pp. 29-47). Springer.

Wakker, P. (1990). Under stochastic dominance Choquet-expected utility and anticipated utility are identical. Theory and Decision, 29, 119-132.

Wakker, P. (2010). Prospect theory. Cambridge University Press.

Wakker, P., \& Deneffe, D. (1996). Eliciting von Newmann-Morgenstern utilities when probabilities are distorted or unknown. Management Science, 42(8), 1131-50.

Wakker, P., \& Tversky, A. (1993). An axiomatization of cumulative prospect theory. Journal of Risk and Uncertainty, 7(2), 147-75.

Weymark, J. (1981). Generalized Gini inequality indices. Mathematical Social Sciences, 1, 409-30.

Yaari, M. (1969). Some remarks on measures of risk aversion and their uses. Journal of Economic Theory, 1, 315-29.

Yaari, M. (1987). The dual theory of choice under risk. Econometrica, 55, 95-115.

Publisher's Note Springer Nature remains neutral with regard to jurisdictional claims in published maps and institutional affiliations. 